\title{
ON THE NUMBER OF REAL ZEROS OF A RANDOM TRIGONOMETRIC POLYNOMIAL
}

BY

\section{SAMBANDHAM}

ABSTRACT. For the random trigonometric polynomial

$$
\sum_{n=1}^{N} g_{n}(t) \cos n \theta
$$

where $g_{n}(t), 0<t<1$, are dependent normal random variables with mean zero, variance one and joint density function

$$
|M|^{1 / 2}(2 \pi)^{-N / 2} \exp \left[-(1 / 2) \bar{a}^{\prime} M \bar{a}\right]
$$

where $M^{-1}$ is the moment matrix with $\rho_{i j}=\rho, 0<\rho<1, i \neq j, i, j=1$, $2, \ldots, N$ and $\bar{a}$ is the column vector, we estimate the probable number of zeros.

1. Consider the random trìgonometric polynomial

$$
\phi(\theta) \equiv \phi(t, \theta)=\sum_{n=1}^{N} g_{n}(t) \cos n \theta,
$$

where $g_{n}(t), 0 \leqslant t \leqslant 1$, are dependent normal random variables with mean zero, variance one and joint density function

$$
|M|^{1 / 2}(2 \pi)^{-n / 2} \exp \left[-(1 / 2) \bar{a}^{\prime} M \bar{a}\right],
$$

where $M^{-1}$ is the moment matrix with $\rho_{i j}=\rho, 0<\rho<1, i \neq j, i, j=1$, $2, \ldots, N$, and $\bar{a}$ is the column vector whose transpose is $\bar{a}^{\prime}=$ $\left(g_{1}(t), \ldots, g_{N}(t)\right)$. In this paper we calculate the probable number of zeros of (1.1). We prove the following.

THEOREM 1. In the interval $0 \leqslant \theta \leqslant 2 \pi$ all save certain exceptional set of functions $\phi(\theta)$ have

$$
\left(2 N / 3^{1 / 2}\right)+O\left(N^{11 / 13+e_{1}}\right)
$$

zeros, when $N$ is large. The measure of the exceptional set does not exceed $N^{-2 \varepsilon_{1}}$, where $\varepsilon_{1}$ is any positive number less than $1 / 13$.

The particular case when $\rho=0$, that is the case when $g_{n}(t)$ are independent normal random variables, was considered by Dunnage [3] and proved the following.

Received by the editors May 26, 1975 and, in revised form, July 19, 1976.

AMS (MOS) subject classifications (1970). Primary 60-XX.

- American Mathematical Society 1978 
THEOREM. In the interval $0 \leqslant \theta \leqslant 2 \pi$ all save a certain exceptional set of the functions $\phi(\theta)$ have

$$
\left(2 / 3^{1 / 2}\right) N+O\left(N^{11 / 13}(\log N)^{3 / 13}\right)
$$

zeros when $N$ is large. The measure of the exceptional set does not exceed $(\log N)^{-1}$.

Das [1] took the class of polynomials

$$
\sum_{n=1}^{N} n^{p}\left(g_{2 n-1} \cos n \theta+g_{2 n} \sin n \theta\right)
$$

where $g_{n}$ are independent normal random variables for a fixed $p>-1 / 2$ and proved the following

THEOREM. In the interval $0 \leqslant \theta \leqslant 2 \pi$, the functions (1.4) have

$$
2[(2 p+1) /(2 p+3)]^{1 / 2} N+O\left(N^{11 / 13+4 q / 13+\varepsilon_{1}}\right)
$$

zeros when $N$ is large. Here $q=\max (0,-p)$ and $\varepsilon_{1}<(2 / 13)(1-2 q)$. The measure of the exceptional set does not exceed $N^{-2 \varepsilon_{1}}$.

Equation (1.4) with $p=0$ has been studied by Clifford Qualls [5] when the random variables are independent and normally distributed with mean zero and variance one. Using the somewhat more advanced techniques developed for stationary Gaussian processes it is shown that the Das result holds for this case also; indeed he is able to reduce the $O$ term to $O\left(N^{3 / 4}(\log N)^{1 / 2}\right)$.

Estimating the average number of real zeros of random trigonometric polynomial is the other interesting topic connected with this type of problems. Das [2] took the trigonometric polynomial of the form

$$
\sum_{n=1}^{N} g_{n} b_{n} \cos n \theta
$$

where $g_{n}$ are independent normal random variables and $b_{n}$ are positive constants. Das proved that the average number of real zeros of $(1.5)$ in $(0,2 \pi)$ for $b_{n}=n^{p}(p \geqslant-1 / 2)$ is

$$
[(2 p+1) /(2 p+3)]^{1 / 2} 2 N+o(N)
$$

and of order $N^{p+3 / 2}$ if $-(3 / 2)<p<-(1 / 2)$, for large $N$.

Sambandham [6] assumed that $g_{n}$ are independent and uniformly distributed in $(-1,1)$ and showed that the average number of real zeros of $(1.5)$ in $(0,2 \pi)$ for $b_{n}=n^{p}(p<-1 / 2)$ and large $N$ is $[(2 p+1) /(2 p+3)]^{1 / 2} 2 N+$ $o(N)$.

When $g_{n}$ are dependent normal random variables and satisfying the condition (1.2) Sambandham [7] showed that the average number of real 
zeros of $(1.5)$ for $b_{n}=n^{p}(p \geqslant 0)$ and large $N$ is

$$
[(2 p+1) /(2 p+3)]^{1 / 2} 2 N+o(N) .
$$

To prove Theorem 1 we follow the procedure adopted by Dunnage and only point out the places where changes are essential.

2. To avoid repetitions we choose the notations of Dunnage. If $\phi(a) \phi(b) \leqslant$ 0 we say that $\phi$ has a single cross over (s.c.o.) in $(a, b)$. In this case $\phi$ has at least one zero in $(a, b)$. Further (i) if $\phi(a) \geqslant 0, \phi(a+b / 2) \leqslant 0$ and $\phi(b) \geqslant 0$ or (ii) if $\phi(a) \leqslant 0, \phi(a+b / 2) \geqslant 0$ and $\phi(b) \leqslant 0$ we say that $\phi$ has double cross over (d.c.o) in $(a, b)$. This can occur only if $\phi$ has at least two zeros in $(a, b)$. Clearly

$$
\operatorname{Pr} \text { (s.c.o. }) \leqslant \operatorname{Pr}(\text { at least one zero) }
$$

and

$$
\operatorname{Pr} \text { (d.c.o. }) \leqslant \operatorname{Pr}(\text { at least two zeros). }
$$

Let

$$
\begin{aligned}
& X_{1}=\mathscr{Q}=\sum_{n=1}^{N} a_{n} g_{n}, \quad X_{2}=\mathscr{P}=\sum_{n=1}^{N} b_{n} g_{n}, \\
& X_{3}=\mathcal{C}=\sum_{n=1}^{N} c_{n} g_{n}, \quad X_{4}=\mathscr{D}=\sum_{n=1}^{N} d_{n} g_{n},
\end{aligned}
$$

where $a_{n}, b_{n}, c_{n}, d_{n}$ are given real numbers and we write

$$
\begin{aligned}
& \gamma_{11}=a^{2}=(1-\rho) \sum_{n=1}^{N} a_{n}^{2}+\rho\left(\sum_{n=1}^{N} a_{n}\right)^{2}, \text { etc., } \\
& \gamma_{12}=(1-\rho) \sum_{n=1}^{N} a_{n} b_{n}+\rho\left(\sum_{n=1}^{N} a_{n}\right)\left(\sum_{n=1}^{N} b_{n}\right), \text { etc. }
\end{aligned}
$$

The characteristic function of the random vector $\left(X_{1}, \ldots, X_{k}\right)(k \leqslant 4)$ is

$$
f\left(t_{1}, \ldots, t_{k}\right)=\exp \left[\left(-\frac{1}{2}\right) \sum_{r, s=1}^{k} \gamma_{r s} t_{r} t_{s}\right]
$$

As Dunnage has indicated in [3] each $g_{n}=g_{n}(t)$ is a continuous function of $t(0 \leqslant t \leqslant 1)$, and hence so are $\mathcal{Q}, \mathscr{B}, \mathcal{C}, \mathscr{D}$. We define $\mu_{1}(t)$ and $\mu_{2}(t)$ by

$$
\mu_{1}(t)=\left[\begin{array}{ll}
1, & \mathscr{Q} \mathscr{B}<0, \\
0, & \mathbb{Q} \mathscr{B}>0,
\end{array} \text { and } \quad \mu_{2}(t)=\left[\begin{array}{ll}
1, & \mathcal{C} \mathscr{D}<0, \\
0, & \mathcal{C} \mathcal{D}>0 .
\end{array}\right.\right.
$$

The functions $\mu_{1}(t)$ and $\mu_{2}(t)$ are measurable and further

$$
\int_{0}^{1} \mu_{1}(t) d t=\operatorname{Pr}(\mathscr{Q} \mathscr{B} \leqslant 0), \quad \int_{0}^{1} \mu_{2}(t) d t=\operatorname{Pr}(\mathcal{C} \mathscr{D} \leqslant 0)
$$


and

$$
\int_{0}^{1} \mu_{1}(t) \mu_{2}(t) d t=\operatorname{Pr}(\mathscr{B} \leqslant 0, \mathcal{C D} \leqslant 0) .
$$

Proceeding by the method of Dunnage we get

(i)

$\operatorname{Pr}(\mathscr{Q}>0, \mathscr{B} \leqslant 0, \mathcal{C}>0)=\operatorname{Pr}(\mathscr{Q}>0, \mathscr{B}>0, \mathcal{C}<0)$

$$
\begin{aligned}
=\frac{1}{4 \pi}\left[\sin ^{-1}\left(1-\frac{\gamma_{23}^{2}}{b^{2} c^{2}}\right)^{1 / 2}-\sin ^{-1}\left(1-\frac{\gamma_{13}^{2}}{a^{2} c^{2}}\right)^{1 / 2}\right. & \\
& \left.+\sin ^{-1}\left(1-\frac{\gamma_{12}^{2}}{a^{2} b^{2}}\right)^{1 / 2}\right],
\end{aligned}
$$

(ii)

$$
\int_{0}^{1} \mu_{1}(t) d t=\frac{1}{\pi} \sin ^{-1}\left(1-\frac{\gamma_{12}^{2}}{a^{2} b^{2}}\right)^{1 / 2}
$$

(iii)

$$
\int_{0}^{1} \mu_{2}(t) d t=\frac{1}{\pi} \sin ^{-1}\left(1-\frac{\gamma_{34}^{2}}{c^{2} d^{2}}\right)^{1 / 2},
$$

(iv)

$$
\begin{aligned}
\int_{0}^{1} \mu_{1}(t) \mu_{2}(t) d t= & -\frac{1}{4}+\frac{1}{2 \pi} \sin ^{-1}\left(1-\frac{\gamma_{12}^{2}}{a^{2} b^{2}}\right)^{1 / 2} \\
& +\frac{1}{2 \pi} \sin ^{-1}\left(1-\frac{\gamma_{34}^{2}}{c^{2} d^{2}}\right)^{1 / 2} \\
& +\frac{1}{2 \pi^{4}} \iiint \int_{-\infty}^{\infty} \frac{\Delta f\left(t_{1}, \ldots, t_{4}\right)}{t_{1} t_{2} t_{3} t_{4}} d t_{1} \ldots d t_{4}
\end{aligned}
$$

where $\Delta\left(f\left(t_{1}, \ldots, t_{4}\right)\right)=\Delta_{2}\left(\Delta_{3}\left(\Delta_{4} f\left(t_{2}, \ldots, t_{4}\right)\right)\right)$ with $\Delta_{r} \psi\left(t_{1}, \ldots, t_{r}, \ldots\right) \equiv$ $\psi\left(t_{1}, \ldots, t_{r}, \ldots\right)-\psi\left(t_{1}, \ldots,-t_{r}, \ldots\right)$ and $f\left(t_{1}, \ldots, t_{4}\right)$ denoting the characteristic function of $(Q, \mathscr{B}, \mathcal{C}, \mathcal{D})$.

3. We divide the zeros of $\phi(\theta)$ into two groups (i) those lying in the intervals $(0, \varepsilon),(\pi-\varepsilon, \pi+\varepsilon)$ and $(2 \pi-\varepsilon, 2 \pi)$ and (ii) those lying in the intervals $(\varepsilon, \pi-\varepsilon)$ and $(\pi+\varepsilon, 2 \pi-\varepsilon)$. The zeros (i) are negligible. We prove this in $\S 6$. To estimate the type (ii) zeros we proceed like that of Dunnage.

We choose $\varepsilon \preceq N^{-3 / 13}$. This choice of $\varepsilon$ is very important. Here by $\lambda(N)$ 
$\measuredangle \nu(N)$ we mean that $\lambda=O(\nu)$ and $\nu=O(\lambda)$. If we restrict $\theta$ to the intervals $\varepsilon<\theta \leqslant \pi-\varepsilon$ and $\pi+\varepsilon \leqslant \theta \leqslant 2 \pi-\varepsilon$ then $\left|\sum_{n=1}^{N} \cos 2 n \theta\right|<(\sin \varepsilon)^{-1}$ and we have $\sum_{n=1}^{N} \cos 2 n \theta=O(1 / \varepsilon)=O\left(N^{3 / 13}\right)$. Similarly we have the following:

$$
\begin{aligned}
\sum_{n=1}^{N} \cos ^{2} n \theta & =\frac{N}{2}+O\left(N^{3 / 13}\right), \\
\sum_{n=1}^{N} n^{2} \sin ^{2} n \theta & =\frac{N^{3}}{6}+O\left(N^{29 / 13}\right), \\
\sum_{n=1}^{N} \cos n \theta & =O\left(N^{3 / 13}\right), \\
\sum_{n=1}^{N} n \sin n \theta & =O\left(N^{16 / 13}\right), \\
\sum_{n=1}^{N} n \sin n \theta \cos n \theta & =O\left(N^{16 / 13}\right) .
\end{aligned}
$$

Let us take $\delta=O\left(N^{-1}\right)$ and put

$$
\mathscr{Q}=\phi(\theta), \quad \mathscr{B}=\phi(\theta+(\delta / 2)), \quad \mathcal{C}=\phi(\theta+\delta) .
$$

We estimate the probability that $\phi$ has a d.c.o. in the interval $(\theta, \theta+\delta)$. This is only double the expression in (2.1). To compute this we need the following:

$$
\begin{aligned}
a^{2}= & (1-\rho) \sum_{n=1}^{N} \cos ^{2} n \theta+\rho\left(\sum_{n=1}^{N} \cos n \theta\right)^{2} \\
b^{2}= & (1-\rho) \sum_{n=1}^{N} \cos ^{2} n\left(\theta+\frac{\delta}{2}\right)+\rho\left(\sum_{n=1}^{N} \cos n\left(\theta+\frac{\delta}{2}\right)\right)^{2} \\
c^{2}= & (1-\rho) \sum_{n=1}^{N} \cos ^{2} n(\theta+\delta)+\rho\left(\sum_{n=1}^{N} \cos n(\theta+\delta)\right)^{2} \\
\gamma_{13}= & (1-\rho) \sum_{n=1}^{N} \cos n \theta \cos n(\theta+\delta) \\
& +\rho\left(\sum_{n=1}^{N} \cos n \theta\right)\left(\sum_{n=1}^{N} \cos n(\theta+\delta)\right) \\
\gamma_{23}= & (1-\rho) \sum_{n=1}^{N} \cos n\left(\theta+\frac{\delta}{2}\right) \cos n(\theta+\delta) \\
& +\rho\left(\sum_{n=1}^{N} \cos n\left(\theta+\frac{\delta}{2}\right)\right)\left(\sum_{n=1}^{N} \cos n(\theta+\delta)\right) .
\end{aligned}
$$


Now we use Taylor's theorem to expand $\left(a^{2} c^{2}-\gamma_{13}^{2}\right)^{1 / 2} / a c$. Suppose that

$$
a^{2} c^{2}-\gamma_{13}^{2}=\alpha_{0}+\alpha_{1} \delta+\alpha_{2}\left(\delta^{2} / 2\right)+\alpha_{2}\left(\delta^{3} / 6\right)+\alpha_{4}\left(\delta^{4} / 24\right) .
$$

It is not difficult to show that (showed in the Appendix)

$$
\begin{aligned}
& \alpha_{0}=\alpha_{1}=0, \\
& \alpha_{2}=(1-\rho)^{2}\left(N^{4} / 6\right)\left[1+O\left(N^{-7 / 13}\right)\right], \\
& \alpha_{3}=O\left(N^{5}\right), \quad \alpha_{4}=O\left(N^{6}\right) .
\end{aligned}
$$

Therefore

$$
\begin{aligned}
\left(a^{2} c^{2}-\gamma_{13}^{2}\right)^{1 / 2} & =\delta\left(\alpha_{2} / 2\right)^{1 / 2}\left(1+\alpha_{3} \delta / 3 \alpha_{2}+O\left(N^{6} \delta^{2} / \alpha_{2}\right)\right)^{1 / 2} \\
& =\delta\left(\alpha_{2} / 2\right)^{1 / 2}\left(1+\alpha_{3} \delta / 6 \alpha_{2}+O\left(N^{2} \delta^{2}\right)\right)
\end{aligned}
$$

Since

$$
\frac{1}{c}=\frac{1}{a}\left(1-\frac{\delta z(\theta)}{a^{2}}+O\left(N^{2} \delta^{2}\right)\right)
$$

where

$$
z(\theta)=2(1-\rho) \sum_{n=1}^{N} n \cos n \theta \sin n \theta+2 \rho\left(\sum_{n=1}^{N} \cos n \theta\right)\left(\sum_{n=1}^{N} n \sin n \theta\right)
$$

we get

$$
\left(1-\frac{\gamma_{13}^{2}}{a^{2} c^{2}}\right)^{1 / 2}
$$

$$
=\left(\delta / a^{2}\right)\left(\alpha_{2} / 2\right)^{1 / 2}\left[1+\left(\frac{\alpha_{3}}{6 \alpha_{2}}-\frac{z(\theta)}{a^{2}}\right) \delta+O\left(N^{2} \delta^{2}\right)\right] .
$$

Similar reasons will lead to

$$
\begin{aligned}
& \left(1-\frac{\gamma_{12}^{2}}{a^{2} b^{2}}\right)^{1 / 2} \\
& \quad=\frac{\delta}{2 a^{2}}\left(\alpha_{2} / 2\right)^{1 / 2}\left[1+\left(\frac{\alpha_{3}}{6 \alpha_{2}}-\frac{z(\theta)}{a^{2}}\right)(\delta / 2)+O\left(N^{2} \delta^{2}\right)\right]
\end{aligned}
$$

and

$$
\begin{aligned}
& \left(1-\frac{\gamma_{23}^{2}}{b^{2} c^{2}}\right)^{1 / 2} \\
& =\left(\delta / 2 a^{2}\right)\left(\alpha_{2} / 2\right)^{1 / 2}\left[1+\left(\frac{\alpha_{3}}{4 \alpha_{2}}-\frac{3 z(\theta)}{2 a^{2}}\right) \delta+O\left(N^{2} \delta^{2}\right)\right]
\end{aligned}
$$


LEMMA 1. If the interval $(\theta, \theta+\delta)$ lies outside the $\varepsilon$-neighbourhoods of $0, \pi$, $2 \pi$ and if $\delta=o\left(N^{-1}\right)$, the probability that $\phi(\theta)$ has a double cross over in this interval is $O\left(N^{3} \delta^{3}\right)$.

Proof. As $x \rightarrow 0, \operatorname{Sin}^{-1} x=x+O\left(x^{3}\right)$. Since $\alpha_{2}=O\left(N^{4}\right), a^{2}=O(N)$, and (3.4), (3.5) and (3.6) are each $O(N \delta)$ and therefore $o(1)$ as $N \rightarrow \infty$. Now proceeding as in [3, Lemma 6$]$ and using the result that the required value is twice the value of $(2.1)$ we get the proof.

If $G$ is any interval of length $\delta=o\left(N^{-1}\right)$ which does not overlap any of the $\varepsilon$-neighbourhoods of $0, \pi, 2 \pi$, let $N(t)$ be the number of zeros that $\phi(\theta, t)$ has in 9 . Here the zeros are counted according to their multiplicity. Further let

$$
N^{*}(t)=\left[\begin{array}{ll}
N(t) & \text { if } N(t) \geqslant 2 \\
0 & \text { otherwise. }
\end{array}\right.
$$

Lemma 2. $\int_{0}^{1} N^{*}(t) d t=O\left(N^{3} \delta^{3}\right)$.

PROOF. If we show that the probability that there being any multiple zeros is zero, then the proof of this lemma follows along the lines of Dunnage's paper [3, Lemmas 11 and 12].

To show this we adopt the method of Dunnage [4, Theorem 3]. If $\phi$ has a multiple zero, real or complex, then for some $\theta, \phi(\theta)=\phi^{\prime}(\theta)=0$ and the elimination of $\theta$ leads to a condition

$$
F\left(g_{1}, g_{2}, \ldots, g_{n}\right)=0
$$

where $F$ is a polynomial in the $g_{n}$. This means that the $N$-dimensional random variables $\left(g_{1}, \ldots, g_{n}\right)$ must lie on a certain space $S$ in the space $R_{n}$. Now this random variable has a density function $\Phi$, say, and the probability that the random variables be on $S$ is

$$
\int_{S} \Phi\left(x_{1}, \ldots, x_{N}\right) d x_{1} \cdots d x_{N}
$$

which is zero since the $N$-dimensional Lebesgue measure of $S$ is zero.

Hence the proof of the lemma.

4. We now divide the interval $(\varepsilon, \pi-\varepsilon)$ into equal subintervals of length $\delta$ and estimate the probability that $\phi(\theta)$ shall simultaneously have a single cross over in each of a given pair of these $\delta$-intervals.

Let

$$
\lambda=N^{14 / 39}, \quad K=N^{1 / 3} \lambda, \quad \delta=o\left(N^{-1} \lambda^{-3 / 7}\right)
$$

and $(\theta, \theta+\delta),(\theta+\tau, \theta+\tau+\delta)$ be two of the $\theta$-intervals into which $(\varepsilon, \pi-\varepsilon)$ is divided so that $\tau>K \delta$. Let

$$
\mathscr{Q}=\phi(\theta), \quad \mathscr{B}=\phi(\theta+\delta), \quad \mathcal{C}=\phi(\theta+\tau), \quad \mathscr{D}=\phi(\theta+\tau+\delta) .
$$

Then $\gamma_{13}=O\left(\varepsilon^{-1}\right)=O\left(K^{-1} \delta^{-1}\right)$. Since $a^{2} \asymp N, c^{2} \asymp N$, we get 


$$
\frac{\gamma_{13}}{a c}=O\left(N^{-1} K^{-1} \delta^{-1}\right)=O\left(N^{-1 / 3} \lambda^{-4 / 7}\right) .
$$

Similarly $\gamma_{14} / a d, \gamma_{23} / b c$, and $\gamma_{24} / b d$ satisfy the inequality (4.2).

In $\S 3, \phi(\theta+\delta)$ was denoted by $\mathcal{C}$, but here we use $\mathscr{B}$ for this purpose. For this change of notation, from (3.6)

$$
\begin{aligned}
1-\frac{\gamma_{12}^{2}}{a^{2} b^{2}}= & \frac{\alpha_{2} \delta^{2}}{2 a^{4}}[1+O(N \delta)] \\
& \asymp N^{2} \delta^{2} \asymp \lambda^{-6 / 7}
\end{aligned}
$$

where $\lambda(N) \asymp v(N)$ denotes that $\lambda=O(v)$ and $v=O(\lambda)$. Similarly

$$
1-\frac{\gamma_{34}^{2}}{c^{2} d^{2}} \asymp \lambda^{-6 / 7} \text {. }
$$

Using (4.2), (4.3), (4.4) and [3, Lemma 13] we shall get

$$
\begin{aligned}
\frac{1}{2 \pi^{4}} \iiint \int_{0}^{\infty} \frac{\Delta f\left(t_{1}, \ldots, t_{4}\right)}{t_{1} t_{2} t_{3} t_{4}} d t_{1} d t_{2} d t_{3} d t_{4} \\
=\left\{\frac{1}{4}-\frac{1}{2 \pi}\left[\operatorname{Sin}^{-1}\left(1-\frac{\gamma_{12}^{2}}{a^{2} b^{2}}\right)^{1 / 2}+\operatorname{Sin}^{-1}\left(1-\frac{\gamma_{34}^{2}}{c^{2} d^{2}}\right)^{1 / 2}\right]\right. \\
\left.\quad+\frac{1}{\pi^{2}} \operatorname{Sin}^{-1}\left(1-\frac{\gamma_{12}}{a^{2} b^{2}}\right)^{1 / 2} \operatorname{Sin}^{-1}\left(1-\frac{\gamma_{34}^{2}}{c^{2} d^{2}}\right)^{1 / 2}+O\left(\lambda^{1 / 7} N^{-2 / 3}\right)\right\} .
\end{aligned}
$$

Combining (2.2), (2.3) and (2.4) with (4.5) we get

$$
\int_{0}^{1} \mu_{1}(t) \mu_{2}(t) d t-\int_{0}^{1} \mu_{1}(t) d t \int_{0}^{1} \mu_{2}(t) d t=O\left(N^{-2 / 3} \lambda^{-1 / 7}\right) .
$$

This shows that when two intervals $(\theta, \theta+\delta)$ and $(\theta+\tau, 0+\tau+\delta)$ are widely spaced $(\tau>K \delta)$ then the probabilities that $\phi(\theta)$ has a s.c.o. in each interval, are almost independent.

5. In this section we calculate the number of zeros of $\phi(\theta)$ in the intervals $(\varepsilon, \pi-\varepsilon)$ and $(\pi+\varepsilon, 2 \pi-\varepsilon)$. Since

$$
\phi(\theta+\pi)=\sum_{n=1}^{N}(-1)^{n} g_{n} \cos n \theta
$$

and $\left(g_{1}, \ldots, g_{N}\right)$ and $\left(-g_{1}, \ldots,-g_{N}\right)$ have the same distribution function it is enough if we consider the iterval $(\varepsilon, \pi-\varepsilon)$.

Divide the interval $(\varepsilon, \pi-\varepsilon)$ into intervals $i_{v}$, each of length $\delta\left(\asymp N^{-1} \lambda^{-3 / 7}\right)$ and with each $i_{v}$ we associate a random function $\mu_{v}(t)$ defined as follows. 


$$
\mu_{v}(t)=\left[\begin{array}{ll}
1 & \text { if } \phi(\theta, t) \text { has a s.c.o. in } i_{v}, \\
0 & \text { otherwise. }
\end{array}\right.
$$

This shows that $\mu_{1}(t)$ and $\mu_{2}(t)$ of $\$ 2$ are a typical pair from the arbitrary set $\left\{\mu_{v}(t)\right\}$. Let $m_{v}=\int_{0}^{1} \mu_{v}(t) d t$.

LEMMA 3. $\Sigma_{v} m_{v}=\left(N / 3^{1 / 2}\right)\left(1+O\left(\lambda^{-3 / 7}\right)\right)$.

Proof. By (2.2)

$$
\begin{aligned}
m_{1} & =\frac{1}{\pi} \sin ^{-1}\left(1-\frac{\gamma_{12}^{2}}{a^{2} b^{2}}\right)^{1 / 2} \\
& =\frac{\delta}{\pi}\left(\alpha_{2} / 2 a^{4}\right)^{1 / 2}(1+O(N \delta)) \\
& =\frac{\delta}{\pi}\left(N / 3^{1 / 2}\right)\left(1+O\left(\lambda^{-3 / 7}\right)\right)
\end{aligned}
$$

from (3.5). This is true for all $m_{v}$ and so

$$
\sum_{v} m_{v}=\left(N / \pi 3^{1 / 2}\right)\left(1+O\left(\lambda^{-3 / 7}\right)\right) \sum_{v} \delta .
$$

But $\Sigma_{v} \delta=\pi-2 \varepsilon=\pi+O\left(\lambda^{-3 / 7}\right)$. Hence the lemma.

LEMMa 4. $\int_{0}^{1}\left[\Sigma_{v}\left(\mu_{v}(t)-m_{v}\right)\right]^{2} d t=O\left(N^{4 / 3} \lambda\right)$.

Proof. Same as the proof of [3, Lemma 17].

Let $N_{v}(t)$ be the number of zeros of $\phi(0, t)$ in $i_{v}$ and let

$$
N_{v}^{*}(t)=\left[\begin{array}{ll}
N_{v}(t) & \text { if } N_{v}(t) \geqslant 2 \\
0 & \text { otherwise }
\end{array}\right.
$$

Clearly

$$
0 \leqslant N_{v}(t)-\mu_{v}(t) \leqslant N_{v}^{*}(t)
$$

and so by Lemma 2

$$
\int_{0}^{1} \sum_{v}\left(N_{v}(t)-\mu_{v}(t)\right) d t \leqslant \sum_{v} \int_{0}^{1} N_{v}^{*}(t) d t=O\left(N \lambda^{-6 / 7}\right) .
$$

The total number of zeros of $\hat{\phi}(\theta, t)$ in $(\varepsilon, \pi-\varepsilon)$ is $\Sigma_{v} N_{v}(t)$ and so we prove

LeMma 5. Except for a set of values of $t$ of measure not exceeding $(1 / 4) N^{-2 \varepsilon_{1}}$

$$
\sum_{v} N_{v}(t)=\left(N / 3^{1 / 2}\right)+O\left(N^{11 / 13+\varepsilon_{1}}\right)
$$

where $\varepsilon_{1}<1 / 13$.

Proof. From Lemma 4 we see that outside a set of $t$ of measure at most $(1 / 8) N^{-2 e_{1}}$ 


$$
\left[\sum_{v}\left(\mu_{v}(t)-m_{v}\right)\right]^{2}=O\left(N^{2 \varepsilon_{1}+(4 / 3)} \lambda\right)
$$

That is

$$
\sum_{v}\left(\mu_{v}(t)-m_{v}\right)=O\left(N^{\varepsilon_{1}+(2 / 3)} \lambda^{1 / 2}\right)
$$

and therefore

$$
\sum_{v} \mu_{v}(t)=\left(N / 3^{1 / 2}\right)+O\left(N \lambda^{-3 / 7}\right)+N\left({ }^{\varepsilon_{1}+(2 / 3)} \lambda^{1 / 2}\right)
$$

outside the exceptional set of $t$. Also (5.2) shows that outside another exceptional set also of measure not exceeding $(1 / 8) N^{-2 \varepsilon_{1}}$

$$
\sum_{v} N_{v}(t)=\sum_{v} \mu_{v}(t)+O\left(N^{1+\varepsilon_{1}} \lambda^{-6 / 7}\right)
$$

and hence combining this with (5.3) we get

$$
\begin{aligned}
\sum_{v} N_{v}(t)= & \left(N / 3^{1 / 2}\right)+O\left(N \lambda^{-3 / 7}\right)+O\left(N^{\varepsilon_{1}+(2 / 3)} \lambda^{1 / 2}\right) \\
& +O\left(N^{1+\varepsilon_{1}} \lambda^{-6 / 7}\right) \\
= & \left(N / 3^{1 / 2}\right)+O\left(N^{\varepsilon_{1}+(11 / 13)}\right)
\end{aligned}
$$

outside an exceptional set of $t$ of measure at most $(1 / 4) N^{-2 \varepsilon_{1}}$.

Further the interval $(\pi+\varepsilon, 2 \pi-\varepsilon)$ can be treated in the same way and so it follows that in the intervals $(\varepsilon, \pi-\varepsilon)$ and $(\pi+\varepsilon, 2 \pi-\varepsilon)$ all the functions $\phi(\theta, t)$ outside an exceptional set of measure at most $(1 / 2) N^{-\varepsilon_{1}}$ have

$$
\left(2 N / 3^{1 / 2}\right)+O\left(N^{(11 / 13)+\varepsilon_{1}}\right)
$$

zeros.

6. In this section we show that outside a small exceptional set of values of $t, \phi(\theta, t)$ has a negligible number of zeros in the intervals $(0, \varepsilon),(\pi-\varepsilon, \pi+$ $\varepsilon)$ and $(2 \pi-\varepsilon, 2 \pi)$. By periodicity the number of zeros in $(0, \varepsilon)$ and $(2 \pi-$ $\varepsilon, 2 \pi)$ is same as the number in $(-\varepsilon, \varepsilon)$ and so we confine out discussion to the interval $(-\varepsilon, \varepsilon)$.

We apply Jensen's theorem to the function of complex argument

$$
\phi(z)=\phi(z, t)=\sum_{n=1}^{N} g_{n}(t) \cos n z
$$

The number of real zeros between $\pm \varepsilon$ does not exceed the number in the circle $|z| \leqslant \varepsilon$. Let $n(r)=n(r, t)$ be the number of zeros of $\phi(z, t)$ in $|z|<r$. By Jensen's theorem 


$$
n(\varepsilon) \log 2<\int_{0}^{2 \varepsilon}[n(t) / t] d t=(2 \pi)^{-1} \int_{0}^{2 \pi} \log \left|\frac{\phi\left(2 \varepsilon e^{i \theta}\right)}{\phi(0)}\right| d \theta
$$

assuming $\phi(0) \neq 0$, and thus

$$
n(\varepsilon) \leqslant(2 \pi \log 2)^{-1} \int_{0}^{2 \pi} \log \left|\frac{\phi\left(2 \varepsilon e^{i \theta}\right)}{\phi(0)}\right| d \theta .
$$

Now $\left|\cos \left(2 N \varepsilon e^{i \theta}\right)\right| \leqslant 2 e^{2 N e}$ and so

$$
\left|\phi\left(2 \varepsilon e^{i \theta}\right)\right| \leqslant 2 e^{2 N \varepsilon}\left(\left|g_{1}\right|+\cdots+\left|g_{N}\right|\right) \leqslant 2 N e^{2 N \varepsilon} \max _{n<N}\left|g_{n}\right| .
$$

Now if $\max \left|g_{n}\right|>N$, then $\left|g_{n}\right|>N$ for at least one value of $n \leqslant N$, so that

$$
\begin{aligned}
P\left(\max \left|g_{n}\right|>N\right) & \leqslant \sum_{n=1}^{N} P\left(\left|g_{n}\right|>N\right) \\
& =N(2 / \pi)^{1 / 2} \int_{N}^{\infty} e^{-(1 / 2) t^{2}} d t \leqslant e^{-(1 / 2) N^{2}}
\end{aligned}
$$

Hence $\left|\phi\left(2 \varepsilon e^{i \theta}, t\right)\right| \leqslant 2 N^{2} e^{2 N \varepsilon}$ outside a $t$-set of measure at most $e^{-(1 / 2) N^{2}}$.

The distribution function of $|\phi(0, t)|=\sum_{n=1}^{N} g_{n}(t)$ is

$$
G(x)=\left[\begin{array}{l}
\left(2 / \pi \Lambda^{2}\right)^{1 / 2} \int_{0}^{x} e^{-t^{2} / 2 \Lambda^{2}} d t, \quad x \geqslant 0 \\
0, \quad x<0
\end{array}\right.
$$

where $\Lambda^{2}=(1-\rho) N+\rho(N-1)$. From this we see that $|\phi(0, t)| \geqslant 1$ except for a set of values of $t$ of measure

$$
\left(2 / \pi \Lambda^{2}\right)^{1 / 2} \int_{0}^{1} e^{-t^{2} / 2 \Lambda^{2}} d t<\left(2 / \pi \Lambda^{2}\right)^{1 / 2}
$$

Combining this with (6.1) we get that for all $\theta$,

$$
\left|\frac{\phi\left(2 \varepsilon e^{i \theta}, t\right)}{\phi(0)}\right|<2 N^{2} e^{2 N \varepsilon}
$$

outside an exceptional set of values of $t$ whose measure does not exceed $e^{-N^{2} / 2}+\left(2 / \pi \Lambda^{2}\right)^{1 / 2}$ which itself does not exceed $(1 / 4) N^{-2 \varepsilon_{1}}$, if $N$ is large enough. From (6.1) and (6.3) we see that outside the exceptional set

$$
n(\varepsilon, t)<1+(2 \log N+2 N \varepsilon) \log 2=O\left(N^{10 / 13}\right) \text {. }
$$

This gives an upper bound for the number of zeros of $\phi(\theta, t)$ in $(-\varepsilon, \varepsilon)$. Similar result can be obtained for $(\pi-\varepsilon, \pi+\varepsilon)$.

Combining (5.5) and (6.4) and adding together all the exceptional sets we get the proof of Theorem 1 .

Acknowledgement. I thank Professor G. Sankaranarayanan, Annamalai University and Professor M. Das, F. M. College, Balasore, Orissa, for their 
guidance and C.S.I.R., India for its financial support during the preparation of this paper. I thank the referee for the suggestions and comments.

APPENDIX. Now we show

$$
\begin{gathered}
\alpha_{0}=\alpha_{1}=0, \quad \alpha_{2}=(1-\rho)^{2} \frac{N^{4}}{6}\left[1+O\left(N^{-7 / 13}\right)\right], \\
\alpha_{3}=O\left(N^{5}\right), \quad \alpha_{4}=O\left(N^{6}\right) .
\end{gathered}
$$

We have used these values from $\S 3$ to $\$ 6$ of this paper.

$$
\begin{aligned}
& \alpha_{0}=\left(a^{2} c^{2}-\gamma_{13}^{2}\right)_{\delta=0} \\
& =\left\{\left[(1-\rho) \sum_{n=1}^{N} \cos ^{2} n \theta+\rho s^{2}(\theta)\right]\right. \\
& \times\left[(1-\rho) \sum_{n=1}^{N} \cos ^{2} n(\theta+\delta)+\rho s^{2}(\theta+\delta)\right] \\
& \left.-\left[(1-\rho) \sum_{n=1}^{N} \cos n \theta \cos n(\theta+\delta)+\rho s(\theta) s(\theta+\delta)^{2}\right]\right\}_{\delta=0} \\
& =0 \text {. } \\
& \alpha_{1}=\left[\frac{d}{d \delta}\left(a^{2} c^{2}-\gamma_{13}^{2}\right)\right]_{\delta=0} \\
& =\left\{\left[(1-\rho) \sum_{n=1}^{N} \cos ^{2} n \theta+\rho s^{2}(\theta)\right]\right. \\
& \times\left[-2(1-\rho) \sum_{n=1}^{N} n \cos n(\theta+\delta) \sin n(\theta+\delta)\right. \\
& \left.+2 \rho s(\theta+\delta) s^{\prime}(\theta+\delta)\right] \\
& -2\left[-(1-\rho) \sum_{n=1}^{N} n \cos n \theta \sin n(\theta+\delta)\right. \\
& \left.+\rho s(\theta) s^{\prime}(\theta+\delta)\right] \\
& \left.\times\left[(1-\rho) \sum_{n=1}^{N} \cos n \theta \cos n(\theta+\delta)+\rho s(\theta) s(\theta+\delta)\right]\right\}_{\delta=0} \\
& =0 \text {. }
\end{aligned}
$$




$$
\begin{aligned}
& \alpha_{2}=\left[\frac{d^{2}}{d \delta^{2}}\left(a^{2} c^{2}-\gamma_{13}^{2}\right)\right]_{\delta=0} \\
& =\left\{\left[(1-\rho) \sum_{n=1}^{N} \cos ^{2} n \theta+\rho s^{2}(\theta)\right]\right. \\
& \times\left[2(1-\rho) \sum_{n=1}^{N} n^{2} \sin ^{2} n(\theta+\delta)\right. \\
& -2(1-\rho) \sum_{n=1}^{N} n^{2} \cos ^{2} n(\theta+\delta)+2 \rho\left(s^{\prime}(\theta+\delta)\right)^{2} \\
& +2 \rho s(\theta+\delta) s^{\prime \prime}(\theta+\delta) \\
& -2\left[-(1-\rho) \sum_{n=1}^{N} n \cos n \theta \sin n(\theta+\delta)+\rho s(\theta) s^{\prime}(\theta+\delta)\right]^{2} \\
& -2\left[(1-\rho) \sum_{n=1}^{N} \cos n \theta \cos n(\theta+\delta)+\rho s(\theta) s(\theta+\delta)\right] \\
& \left.\times\left[-(1-\rho) \sum_{n=1}^{N} n^{2} \cos n \theta \cos n(\theta+\delta)+\rho s(\theta) s^{\prime \prime}(\theta+\delta)\right]\right\}_{\delta=0} \\
& =\left[(1-\rho) \sum_{n=1}^{N} \cos ^{2} n \theta+\rho s^{2}(\theta)\right]\left[2(1-\rho) \sum_{n=1}^{N} n^{2} \sin ^{2} n \theta\right. \\
& -2(1-\rho) \sum_{n=1}^{N} n^{2} \cos ^{2} n \theta \\
& \left.+2 \rho\left(s^{\prime}(\theta)\right)^{2}+2 \rho s(\theta) s^{\prime \prime}(\theta)\right] \\
& -2\left[(1-\rho) \sum_{n=1}^{N} n \cos n \theta \sin n \theta+\rho s(\theta) s^{\prime}(\theta)\right]^{2} \\
& -2\left[(1-\rho) \sum_{n=1}^{N} \cos ^{2} n \theta+\rho s^{2}(\theta)\right] \\
& \times\left[-(1-\rho) \sum_{n=1}^{N} n^{2} \cos ^{2} n \theta+\rho s(\theta) s^{\prime \prime}(\theta)\right] \text {. }
\end{aligned}
$$


Using the values in $\$ 3$, we find that only the last product in the above contains the maximum value. That is

$$
\alpha_{2}=(1-\rho)^{2} \frac{N^{4}}{6}\left[1+O\left(N^{-7 / 13}\right)\right] \text {. }
$$

Similarly successive differentiation gives

$$
\alpha_{3}=O\left(N^{5}\right) \text { and } \alpha_{4}=O\left(N^{6}\right) \text {. }
$$

\section{REFERENCES}

1. M. Das, The number of real zeros of a class of random trigonometric polynomials, Math. Student 40A (1972), 305-317. MR 49 \# 1584.

2. Cambridge Philos. Soc. 64 (1968), 721-729. MR 38 \# 1720.

3. J. E. A. Dunnage, The number of real zeros of a random trigonometric polynomial, Proc. London. Math. Soc. (3) 16 (1966), 53-84. MR 33 \# 757.

4. The number of real zeros of a class of random algebraic polynomials, Proc. London Math. Soc. (3) 18 (1968), 439-460. MR 37 \#5903.

5. Clifford Qualls, On the number of zeros of a stationary Gaussian random trigonometric polynomial, J. London Math. Soc. (2) 2 (1970), 216-220. MR 41 \#2757.

6. M. Sambandham, On a random trigonometric polynomial, Indian J. Pure. Appl. Math. (to appear).

7. __ On random trigonometric polynomial, Indian J. Pure. Appl. Math. (to appear).

Department of Mathematics, ANnamalai University, Nagar 608 101, India

Current address: Department of Mathematics, Ayya Nadar Janaki Ammal College, Sivakasi, 626124, India 